Meta

Journal des traducteurs

Translators' Journal

\title{
Comment traduire Rimbaud : problèmes psycholinguistiques
}

\section{Daniel Slote}

Volume 27, numéro 1, mars 1982

Psychanalyse et traduction

URI : https://id.erudit.org/iderudit/004171ar

DOI : https://doi.org/10.7202/004171ar

Aller au sommaire du numéro

Éditeur(s)

Les Presses de l'Université de Montréal

ISSN

0026-0452 (imprimé)

1492-1421 (numérique)

Découvrir la revue

Citer cet article

Slote, D. (1982). Comment traduire Rimbaud : problèmes psycholinguistiques. Meta, 27(1), 129-136. https://doi.org/10.7202/004171ar d'utilisation que vous pouvez consulter en ligne.

https://apropos.erudit.org/fr/usagers/politique-dutilisation/ 


\title{
COMMENT TRADUIRE RIMBAUD :
}

\author{
PROBLÈMES PSYCHOLINGUISTIQUES
}

Daniel Slote

Pour traduire Rimbaud, il est essentiel de comprendre son œuvre. Cette compréhension devrait se fonder sur une appréciation juste de sa poésie en passant par la critique littéraire. Sinon, on risque de négliger une emphase juste, inhérente à l'œuvre rimbaldienne. Les pages qui suivent vont essayer de mettre en relief cette emphase juste, en analysant d'une part les poèmes «de jeunesse» et les poèmes écrits sous l'effet des expériences dites «du voyant» d'autre part.

Cette analyse, nous l'espérons, donnera lieu à une éventuelle théorie bien réfléchie (et non automatique) de la traduction des œuvres de Rimbaud en général et des Illuminations en particulier. Autrement dit, nous espérons que cette appréciation critique de sa poésie nous amènera à réfléchir d'abord sur une théorie axée sur la littérature et sur une théorie axée sur la traduction ensuite.

Or, la pratique de la traduction devient dans ce cas un moyen privilégié d'être sensible à la praxis verbale de Rimbaud. Dans cet espace privilégié, donc, qu'est la traduction, il s'agit de trouver le point stratégique d'où l'on pourrait apercevoir le paysage métaphorique rimbaldien spécifique; ce point où la lecture de l'œuvre en langue étrangère - quelle qu'elle soit - produirait la même réponse affective (Wolfgang Iser et coll.) que celle éprouvée par le lecteur français.

Attachons-nous aux problèmes particuliers que posent les Illuminations. Le principal obstacle, outre certaines difficultés d'interprétation, de lecture et de «sens ${ }^{1}$ se dresse sur le plan sémique spécifique du poète. Il va de soi que chaque écrivain et, à plus forte raison, chaque poète, se meut dans son champ lexical bien déterminé où les mots pris isolément n'ont rien de particulier. Toutefois, il est intéressant de constater avec Ivan Fónagy, Jean-Pierre Richard et d'autres que les éléments qui définissent un champ lexical, pris dans leur ensemble, constituent une aire métaphorique propre à chaque poète. C'est ce champ sémique spécifique qu'il faudrait explorer chez Rimbaud dans le but de dégager ces mots-clefs que l'on retrouve enchâssés dans les Illuminations. S'il est vrai, comme nous le croyons, que le contenu métaphorique de ce champ remonte au tout début de la vie créatrice de Rimbaud, il est essentiel

1. Daniel Slote (1978): «Translating Rimbaud's Illuminations», META, vol. 23, $\mathrm{n}^{\circ}$ 1, mars, Montréal, Presses de l'Université de Montréal, p. 86-96. 
pour le traducteur de compiler un lexique de ces termes, de les traduire, et d'y adhérer dans sa traduction sans avoir recours aux synonymes, équivalents ou variantes comme tout traducteur, dans d'autres circonstances, serait tenté de faire. Bien entendu, les mots constituent la trame même de toute poésie; cependant, chez un poète où «l'alchimie du verbe» était le point de départ de tout acte métaphorique, il est d'autant plus vital pour le traducteur de cerner ce champ spécifique avec justesse et rigueur.

Or, une fois dégagée, cette configuration sémique doit conserver sa dimension obsessionnelle : la répétition et l'intensité de certains termes chez Rimbaud dénotent que leur présence dans les poèmes n'est pas fortuite ${ }^{2}$. Le traducteur, on le sait, se trouve toujours confronté à des problèmes de répétition qu'il essaie, dans un but souvent louable, de résoudre en les éliminant grâce aux procédés linguistiques bien connus du spécialiste. Néanmoins, ici, face au texte rimbaldien dont la nature intrinsèque consiste en la répétition, le traducteur doit se méfier de sa déformation innée qui le pousse à chercher des synonymes ou des variantes stylistiques : il est essentiel (et si l'on se répète ici, c'est par mimétisme, compte tenu du problème dont il est question...) de ne pas trahir l'aspect compulsif de l'œuvre de Rimbaud, d'où le besoin impératif d'écarter toute paraphrase, toute variante, de cette constellation sémique. Le traducteur doit reproduire les éléments de celle-ci, sous une forme qui suggère, limite, refiète, l'original de par leur répétition, leur fréquence et leur intensité.

Avant Une Saison en Enfer et les Illuminations, le contenu sémique du champ métaphorique de Rimbaud se trouve dispersé dans tous les poèmes «de jeunesse» d'une part, et concentré dans les diverses versions de son «Être de Beauté» d'autre part. Celui-ci trouve son expression la plus complète dans les Illuminations où, avant de s'épanouir dans un mouvement qui transcende le poème pour se résorber enfin dans le Silence, les termes spécifiques les plus importants convergent. Cet Être, ce Génie, constitue l'idéation des principales obsessions de Rimbaud, transposées dans le champ métaphorique spécifique du poète.

Essayons maintenant de dégager les traits sémiques propres à l'œuvre de Rimbaud, ce qui devrait aider le traducteur à établir une constellation verbale à l'instar de celle créée par l'auteur.

L'Être de Beauté revêt de multiples masques tout au long de l'œuvre de Rimbaud, mais certains attributs sont toujours associés à l'Être, soit force (surtout dans les bras), lumière, immensité, musique, couleur, amour, rêve. Certaines épithètes accompagnent l'Être, soit glace, neige, cristal, blessé (sanglant), frisson, les verbes souffler, se dresser, trembler, se détourner (tête) etc. La destruction par le feu ou par l'eau joue un rôle capital dans la création de l'Etre : la vision finale de l'Être se dresse sur les ruines de tout ce que Rimbaud a voulu détruire. Le silence est important dans cette tâche colossale; après tout, c'était l'aboutissement de sa tentative de voler du feu: après que ses visions lui eurent fait défaut, e'est le silence littéraire ${ }^{3}$. Il ne faut pas oublier

2. Voir Charles Mauron et coll.

3. Peu importe s'il s'agit du silence de «l'Adieu» de la Saison ou le silence implicite dans «Solde» des Illuminations. 
l'élément maternel dans la création de l'Être : la fusion fréquente des notions de déesse et de mère.

Le rôle attribué aux anges par Rimbaud, du moins dans ses premiers poèmes, est celui d'un deus ex machina: l'ange de l'Ange et l'Enfant par exemple. Le même phénomène existe dans Les Étrennes: c'est «l'ange des berceaux " qui «met un rêve joyeux» dans leur «lourd sommeil ». Grâce au pouvoir magique de cet ange, l'amour et la joie envahissent l'âme des enfants qui

se croient endormis dans un paradis rose.

On sait que les anges font partie de «l'œuvre inouïe» du Voyant; dans «Matinée d'Ivresse $»$ la vision se révèle sous les traits

des anges de flamme et de glace

et dans «Mystique»:

Les anges tournent leurs robes de laine dans les herbages d'acier et d'émeraude;

par extension, Eucharis dans «Avant le Déluge» est un ange qui annonce à Rimbaud :

c'était le printemps

et même les Normes, ces Parques scandinaves, dans «Soir Historique» jouent le rôle d'un héraut.

Soleil et Chair est un poème sur «l'amour infini» dont Rimbaud avait parlé déjà dans Sensation. Il

regrette les temps de la grande Cybèle,

la magna mater de l'antiquité; elle est «gigantesquement belle» et de

son double sein versait dans les immensités

Le pur ruissellement de la vie infinie,

Rimbaud croit en cette déesse, en cette «divine mère " qui incarne l'amour, «la grande Foi»; Astarté possède des «grands yeux noirs vainqueurs» et c'est dans

son nombril rose où vint neiger l'écume

et Cypris «étrangement belle» montre «son ventre neigeux». Il y a de la musique associée à Astarté :

et fit chanter, Déesse [...]

Le rossignol aux bois et l'amour dans les cœurs.

Tout est grand dans le poème :

le monde vibrera comme une immense lyre,

même les baisers sont immenses :

le frémissement d'un immense baiser;

Héraclès a «un vaste corps»; les astres sont un «immense troupeau» se déplaçant dans «l'éther vaste»; la «Mère-Nature» possède un «immense creu- 
set» et l'homme se trouve «dans l'immense splendeur de la riche nature». L'allusion à Zeus est importante :

il tourne lentement vers elle son œil vague.

Le génie dans « $\AA$ Une Raison» accomplit «le nouvel amour» par un geste semblable :

Ta tête se détourne : le nouvel amour! Ta tête se retourne, - le nouvel amour!

et dans «Génie» Rimbaud parle de «ses têtes» et de «sa vue».

Rimbaud s'identifie à Ophélie dans le poème du même nom; comme à l'esprit de celle-ci, un souffle

à (son) esprit rêveur portait d'étranges bruits

et son cour aussi «écoutait le chant de la Nature». Il ne faut pas oublier qu'Ophélia est morte folle; de cette folie que Rimbaud décrit ainsi :

Tu te fondais à lui (à Hamlet) comme une neige au feu

et les épithètes soulignent sa blancheur : «la blanche Ophélia» (à deux reprises) et aussi : "Ô pâle Ophélia, belle comme la neige»; elle est comme un "fantôme blanc» et elle "flotte comme un grand $l y s$ ». La marge entre les déesses décrites dans Soleil et Chair et Ophélie elle-même est très mince; pourtant au lieu d'être "gigantesque", Ophélie est frêle et tombe victime de la Nature qui «brisait (son) sein d'enfant». Si elle n'est point déesse, elle flotte sur des eaux semblables à celles d'où sortit Vénus. La musique l'accompagne dans la mort :

un chant mystérieux tombe des astres d'or.

Sa mort même ressemble étrangement à une naissance car Rimbaud, «le Poète » de la dernière strophe la voit venir «chercher, la nuit, les fleurs».

Le Forgeron, ce symbole de la liberté, rappelle les dieux ou les héros décrits par Rimbaud: Hercule et Zeus. Comme eux, le forgeron est immense : il a «le front vaste» et des "énormes épaules» et dans son immensité il est "effrayant d'ivresse et de grandeur»; son marteau, symbole double de destruction et de construction est à sa taille : «le bras sur un marteau gigantesque». Tout comme celui du Génie, son regard est puissant :

prenant ce gros-là dans son regard farouche, le forgeron détruit le roi à coups de paroles, mais cette destruction est nécessaire si «le nouvel amour» ("À Une Raison») doit venir sur la terre :

[...] Nos marteaux en main, passons au crible

Tout ce que nous savons : puis, Frères, en avant!

Le forgeron parle des «grands temps nouveaux» où l'homme

domptera les choses

Et montera sur Tout, comme sur un cheval!;

cette vision du forgeron, de ce dieu «superbe de crasse» contient des éléments du «Noël» dont parle Rimbaud dans «Matin» (Saison) : «la naissance du travail nouveau » qui aura lieu à cette époque utopique des «grands temps nouveaux» (Forgeron); nous savons qu'il s'agit d'un rêve puisque le forgeron lui-même nous annonce: "nous faisons quelquefois ce grand rêve». 
Cybèle, bien sûr, a son antipode: Clara Vénus Anadyomène qui est «belle hideusement d'un ulcère à l'anus »; on remarquera que son grand corps représente le seul point commun avec les vraies déesses de Soleil et Chair: «sa large croupe». On ne peut concevoir pleinement le beau sans connaître son contraire, et Rimbaud décrit une Vénus horriblement humaine qui sort non point de l'onde mais «d'une vieille baignoire». Il s'agit des mêmes Vénus, les «laiderons» de Mes Petites Amoureuses que Rimbaud rejette si violemment.

Les attributs de l'Être déjà vus se multiplient et se concentrent au cours de la période entre 1863 et 1873 et peut-être au-delà. On vient de voir l'admiration de Rimbaud pour l'immensité physique dans ses premiers poèmes et on constate que cette admiration ne cesse de croitre pendant sa période de voyance.

Dans Oraison du Soir, il empoigne "une chope à fortes cannelures»; «l'air est gonflé d'impalpables voilures». Les mains de Jeanne-Marie sont «plus fortes que tout un cheval» et «plus fatales que des machines». Il décrit Jeanne-Marie, symbole de la liberté semblable au Forgeron, en des termes qui rappellent sa façon de décrire des déesses : elle a des «mains d'ange» et ses mains sont associées à l'amour :

Elles ont pâli, merveilleuses,

Au grand soleil d'amour chargé

et dans la strophe écrite de la main de Verlaine :

l'éclat de ces mains amoureuses

Tourne le crâne des brebis!

Ces mains sont capables de grands rêves :

Oh quel Rêve les a saisis

Dans les pandiculations!

Un rêve inouï des Asies

Des Khenghavars ou des Sions?

Le désert «où luit la Liberté ravie»dans Les Poètes de Sept Ans est un «grand désert» et ses rêves deviennent de plus en plus immenses :

Il rêvait la prairie amoureuse, où des houles

Lumineuses, parfums sains, pubescences d'or,

Font leur remuement calme et prennent leur essor!

Ces mêmes rêves immenses se trouvent dans Les Saurs de Charité : Rimbaud parle des «vastes fins, Rêves ou Promenades immenses» et la Femme qui est un «monceau d'entrailles» possède «d'immenses prunelles»; dans «Le Juste... » la vision cosmique est gigantesque :

Tu veux voir rutiler les bolides?

Et, debout, écouter bourdonner les fleurs

D'astres lactés, et les essaims d'astéroïdes?

et

l'ordre, éternel veilleur [...] (fait un) remuement énorme sans désastres.

Dans Ce qu'on dit au Poète à propos des Fleurs il parle des fleurs gigantesques que 
ni Renan, ni le chat Murr

N'ont vu;

il s'agit des «Bleus Thyrses immenses».

Dans Bateau Ivre Rimbaud a vu «fermenter les marais énormes [...] où pourrit [...] tout un Léviathan» et il tremblait

sentent geindre à cinquante lieues

Le rut des Béhémots, et les Maelstroms épais;

tout est grand dans le poème: il suffit de comparer «des archipels sidéraux» qu'il a vus dans ses visions à la «flache noire et froide» qui se trouve en Europe, dans le réel, pour s'en rendre compte. Ses visions sont souvent accompagnées de frissons :

Les flots roulant au loin leur frisson de volets;

il a rêvé «aux neiges éblouies»; il parle des "pieds lumineux des Maries» et dans toute une série d'images, la blancheur assume une importance capitale :

Glaciers, soleils d'argent, flots nacreux, cieux de braises;

la mer elle-même est «lactescente» et sur cette mer flottent les bateaux transportant du «coton anglais» que Rimbaud regrette dans la dernière strophe du poème: «Je ne puis plus [...] enlever leur sillage aux porteurs de cotons». Le voyage fabuleux a eu lieu "l'autre hiver» moment choisi par Rimbaud pour retrouver la chaleur aux «incroyables Florides".

Même dans Larme le froid fait partie de sa vision:

Le vent, du ciel, jetait des glaçons aux mares;

dans La Rivière de Cassis les corbeaux ont des «voix d'anges»; Vénus revient dans Comédie de la Soif («l'Esprit»): «Vénus, scur de l'azur»; et aux «Juifs errants de Norvège» Rimbaud demande : «Dites-moi la neige». Vénus est priée dans Bonne Pensée du Matin de laisser "un peu les Amants» pour porter

aux travailleurs l'eau de vie,

Pour que leurs forces soient en paix.

Rimbaud bâtit son Être dans l'état de Voyance exactement comme un sculpteur fait surgir la forme de l'informe: il parle, dans «Being Beauteous» de «la Vision, sur le chantier» et dans «Matinée d'Ivresse» son œuvre se dessine devant ses yeux sur son «chevalet féerique»:

Hourra pour l'œuvre inouïe et pour le corps merveilleux pour la première fois !

Les mêmes éléments de construction se trouvent dans le poème: la musique créatrice et «atroce» parce qu'elle exige l'effort immense du Voyant, sonne comme une ouverture :

Fanfare atroce où je ne trébuche point!

C'est la fanfare du clairon et du devoir qu'entendit le Forgeron et qui sonna dans les rues sanglantes de Paris insurgé. 
L'innocence des «rires d'enfants» accompagne l'éclosion de la Vision, sorte de musique pure dont Rimbaud a déjà parlé, et joue un rôle important dans la méthode :

cela commença sous les rires des enfants, cela finira par eux.

Cette hallucination de l'ouie contraste avec les bruits du réel :

ce poison va rester dans toutes nos veines même

quand, la fanfare tournant, nous serons rendus

à l'ancienne inharmonie.

L'Être accomplit tout: dans cette «débandade de parfums» de «Matinée d'Ivresse " tout est beau :

horreur des figures et des objets d'ici, sacrés

soyez-vous par le souvenir de cette veille;

il transforme «la rustrerie» en «anges de flamme et de glace».

Rimbaud a travaillé dur pour atteindre la Vision de l'Être. Il nous en parle dans plusieurs Illuminations : dans «Jeunesse IV» il se dit:

tu te mettras à ce travail : toutes les possibilités harmoniques et architecturales s'émouvront à tes expériences

et cette phrase capitale :

Ta mémoire et tes sens ne seront que la nourriture de ton impulsion créatrice.

Lorsqu'il met ses «calculs» de côté c'est pour écouter sa mémoire :

la visite des souvenirs et la séance des rythmes occupent la demeure, la tête et le monde de l'esprit;

aussitôt après il reprend

l'étude au bruit de l'œuvre dévorante qui se rassemble et remonte dans les masses.

Dans «Guerre» il parle de nouveau de ses calculs :

à présent l'inflexion éternelle des moments et l'infini des mathématiques me chassent par ce monde où je subis tous les succès civils, respecté de l'enfance étrange et des affections énormes.

Dans le poème il songe à une guerre qui amènerait l'Être sur la terre, une guerre de droit ou de force, de logique bien imprévue,

une guerre qui est «aussi simple qu'une phrase musicale».

C'est surtout dans «Vies II» que Rimbaud dresse le bilan de ses talents d'inventeur :

Je suis un inventeur bien autrement méritant que tous ceux qui m'ont précédé : un musicien même, qui ai trouvé quelque chose comme la clef de l'amour.

Pour compléter l'image de l'Être il importe de décrire ses gestes : «le nouvel amour» que l'Être apporte au monde dans «À Une Raison» s'accomplit par un geste dont nous avons vu l'importance :

Ta tête se détourne : le nouvel amour! Ta tête se retourne, - le nouvel amour! 
Un simple pas de l'Être fait des miracles :

c'est la levée des nouveaux hommes et leur en marche;

il est prié :

Change nos lots, crible les fléaux, à commencer par le temps

et on lui supplie d'élever

n'importe où la substance de nos vœux.

Un autre geste crée «la nouvelle harmonie», la perfection :

Un coup de ton doigt sur le tambour décharge tous les sons et commence la nouvelle harmonie,

et dans son éternel mouvement il n'a ni commencement ni fin :

arrivée de toujours, qui t'en iras partout.

Nous constatons, pour finir, que les attributs de l'Être existent dans l'esprit de Rimbaud depuis son enfance; il ne s'agit donc pas, dans les Illuminations, d'une création toute neuve et de toutes pièces mais plutôt d'une concentration et d'une projection des éléments idéationnels qui étaient là.

C'est au traducteur de respecter soigneusement l'impact de cette idéation émergée, de la reproduire dans sa fréquence et dans son intensité grâce à la répétition littérale des zones métaphoriques. La compilation d'un lexique des termes qui définissent ces zones permettrait au traducteur d'utiliser les élémentsbâtisseurs de l'édifice métaphorique rimbaldien, et ce avec la même insistance obsessionnelle que celle de l'original.

\section{BIBLIOGRAPHIE}

ISER, Wolfgang (1974): The Implied Reader: Patterns of Communication in Prose Fiction from Bunyan to Beckett, Baltimore.

MAURON, Charles (1964): Des métaphores obsédantes au mythe personnel, Paris.

MAURON, Charles (1969): l'Inconscient dans l'ceuvre et la vie de Racine, Paris.

MICHAUD, G. (1961): Message poétique du symbolisme, Paris.

PETITFILS, Pierre (1949) : l'Oeuvre et le visage d'Arthur Rimbaud, Paris.

PITRES, A. et E. REGIS (1902) : les Obsessions et les impulsions, Paris.

RICHARD, Jean-Pierre (1961) : l'Univers imaginaire de Mallarmé, Paris.

RIMBAUD, A. (1971) : les Illuminations, traduites par Daniel Slote, Montréal.

RIMBAUD, A. (1954): Oeuvres complètes, ed., Rolland de Renéville et Jules Mouquet, «Bibliothèque de la Pléiade», Paris.

SLOTE, Daniel (1978): «Translating Rimbaud's Illuminations : Games with Words», Meta, vol. $23, \mathrm{n}^{\circ} 1$, mars. 